Article

\title{
Nine Different Chemical Species and Action Mechanisms of Pancreatic Lipase Ligands Screened Out from Forsythia suspensa Leaves All at One Time
}

\author{
Tinggui Chen *, Yayun Li and Liwei Zhang \\ Institute of Molecular Science, Key Laboratory of Chemical Biology and Molecular Engineering of Ministry of \\ Education, Shanxi University, Taiyuan 030006, China; lisf@sxu.edu.cn (Y.L.); lwzhang@sxu.edu.cn (L.Z.) \\ * Correspondence: chentg@sxu.edu.cn; Tel.: +86-351-7018113
}

Academic Editor: Thomas J. Schmidt

Received: 30 March 2017; Accepted: 10 May 2017; Published: 12 May 2017

\begin{abstract}
It is difficult to screen out as many active components as possible from natural plants all at one time. In this study, subfractions of Forsythia suspensa leaves were firstly prepared; then, their inhibitive abilities on pancreatic lipase were tested; finally, the highest inhibiting subfraction was screened by self-made immobilized pancreatic lipase. Results showed that nine ligands, including eight inhibitors and one promotor, were screened out all at one time. They were three flavonoids (rutin, $\mathrm{IC}_{50}$ : $149 \pm 6.0 \mu \mathrm{mol} / \mathrm{L}$; hesperidin, $52.4 \mu \mathrm{mol} / \mathrm{L}$; kaempferol-3-O-rutinoside, isolated from $F$. suspensa leaves for the first time, $\mathrm{IC}_{50}$ notably reached $2.9 \pm 0.5 \mu \mathrm{mol} / \mathrm{L}$ ), two polyphenols (chlorogenic acid, $3150 \pm 120 \mu \mathrm{mol} / \mathrm{L}$; caffeic acid, $1394 \pm 52 \mu \mathrm{mol} / \mathrm{L}$ ), two lignans (phillyrin, promoter; arctigenin, $2129 \pm 10.5 \mu \mathrm{mol} / \mathrm{L}$ ), and two phenethyl alcohol (forsythiaside A, $2155 \pm 8.5 \mu \mathrm{mol} / \mathrm{L}$; its isomer). Their action mechanisms included competitive inhibition, competitive promotion, noncompetitive inhibition, and uncompetitive inhibition. In sum, using the appropriate methods, more active ingredients can be simply and quickly screened out all at one time from a complex natural product system. In addition, F. suspensa leaves contain numerous inhibitors of pancreatic lipase.
\end{abstract}

Keywords: Forsythia suspensa leaves; affinity screening; pancreatic lipase inhibitor; obesity

\section{Introduction}

Obesity has become one of the greatest threats to human health in this century. It is recognized as the largest global chronic disease by WHO [1,2]. At least 3,400,000 adults die each year as a result of being overweight or obese [3,4]. Because pancreatic lipase (PL) can decompose $50-70 \%$ of fat, developing its inhibitor as an anti-obesity drug is preferred. Orlistat is a type of long-term PL inhibitor found and modified from Streptomyces toxytricini and is currently one of the main drugs for treating obesity. However, this drug produces several side effects including fatty diarrhea, stool urgency, fecal incontinence, allergies, and liver function damage [5-7].

In recent years, developing safer and more effective PL inhibitors from natural compounds has attracted more and more attention [8-11], and some new technologies are emerging [12,13]. We also used immobilized enzyme technology to screen PL inhibitors from Polygonum cuspidatum [14].

Forsythia suspensa (Thunb.) Vahl is widely distributed in China, Japan, and Korea. Their fruits have anti-inflammatory, anti-viral, anti-pyretic, anti-liver injury, and other effects [15-18]. Their leaves are often used for tea. In this study, in order to find out whether F. suspensa tea, like black tea [19], oolong tea [20], and green tea [21], also possess an inhibitory effect on PL, its subfractions were prepared, and their inhibitive ability on PL were detected; then, the highest inhibiting subfraction was screened by self-made immobilized PL. 


\section{Results and Discussion}

\subsection{Inhibition of the Subfractions of F. suspensa Leaves on PL}

As a positive drug, the inhibition rate of $5 \mu \mathrm{g} / \mathrm{mL}$ orlistat reached $58 \%$, and its success proved the feasibility of the enzyme activity assay. The inhibitory effects of crude extracts and various extract subfractions of F. suspensa leaves on PL at $1500 \mu \mathrm{g} / \mathrm{mL}$ are shown in Table 1 . The table shows that the inhibition rate of the remaining parts on PL was the highest and reached $81.48 \%$. Thus, the remaining precipitation of F. suspensa leaves was selected as the screening object of immobilized enzyme.

Table 1. Inhibitory ratio of subfractions of $F$. suspensa leaves on pancreatic lipase $(n=3)$.

\begin{tabular}{cc}
\hline Subfractions $(\mathbf{1 5 0 0} \mu \mathrm{g} / \mathrm{mL})$ & Inhibitory Ratio \\
\hline Crude extract & $39.28 \%$ \\
Acetic ether extract & $44.17 \%$ \\
Ethanol extract & $15.91 \%$ \\
Water extract & $50.21 \%$ \\
Remaining precipitate & $81.48 \%$ \\
\hline
\end{tabular}

\subsection{Screening of PL Inhibitors in the Remaining Precipitation}

The HPLC results are shown in Figure 1. Figure 1A,B shows that 21 absorption peaks occurred in the remaining precipitation. Ten compounds, which may interact with PL, were screened out by the immobilized enzyme (Figure 1C,D).

Among the ten compounds, Compounds 1, 2, 4, 5, 8 and 9 were rutin, phillyrin, chlorogenic acid, caffeic acid, forsythiaside $\mathrm{A}$, and hesperidin respectively by compared with the mixed standard substances (Figure 1E). The undetermined compounds were separated and identified by HPLC-MS/MS; Compounds 6, 7, and 10 were identified as an isomer of forsythiaside A, arctigenin, and kaempferol-3-O-rutinoside, shown in Figure 2 and Table 2. Compound 3 had not been previously identified. The fully identified 8 PL ligands from F. suspensa leaves are shown in Figure 3.

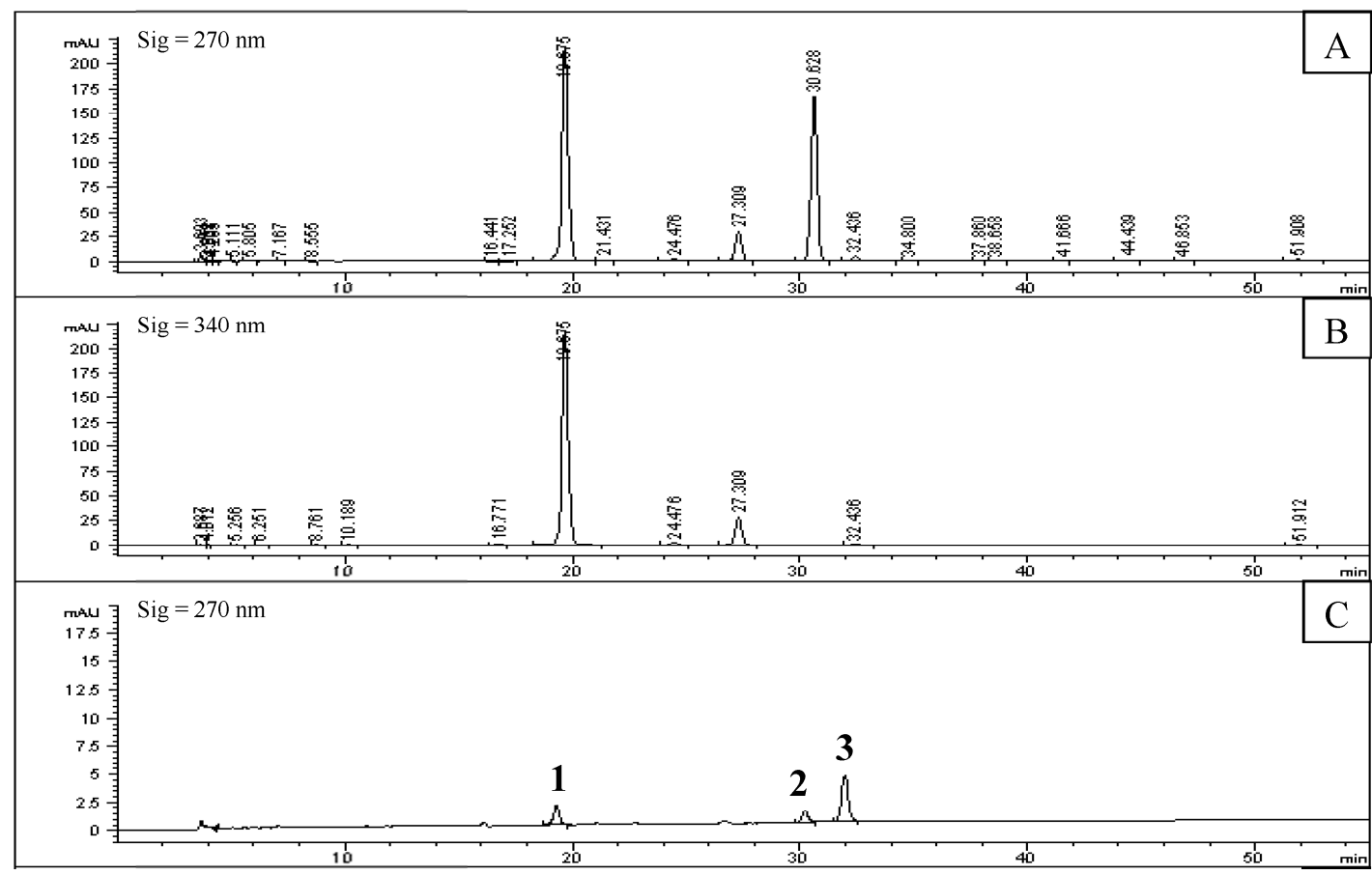

Figure 1. Cont. 


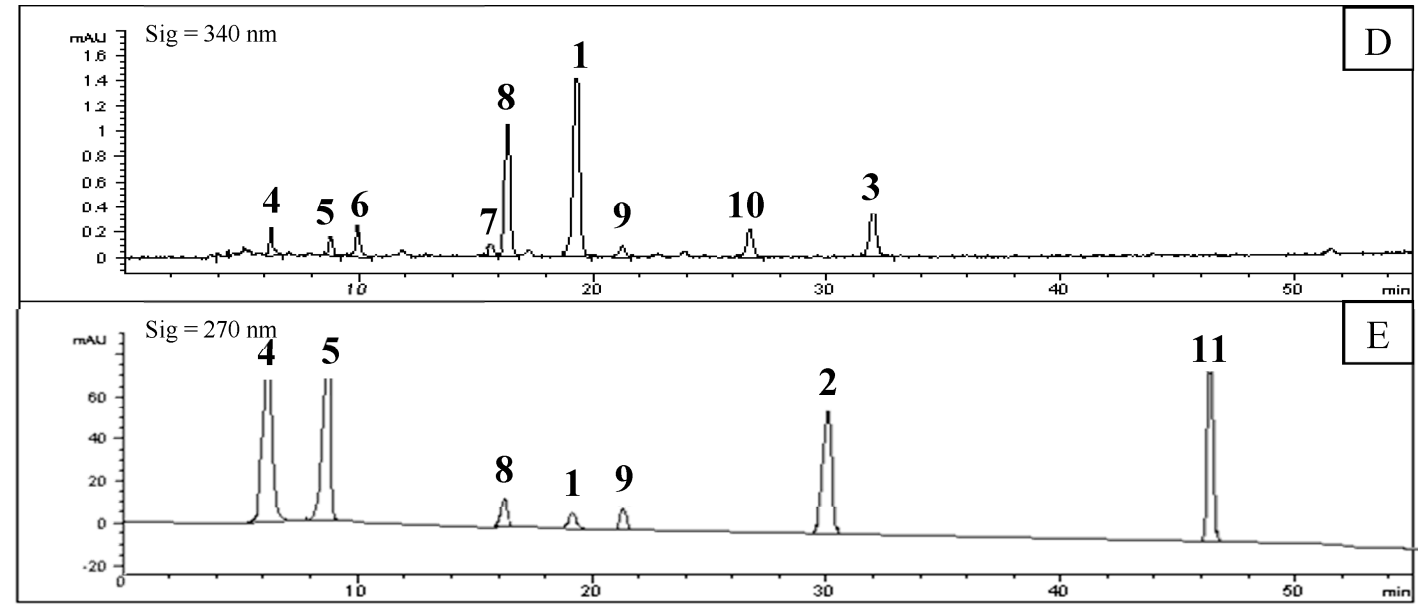

Figure 1. HPLC chromatograms of remaining precipitate of $F$. suspensa leaves (A: $270 \mathrm{~nm} ; \mathbf{B}: 340 \mathrm{~nm})$, solution of screened out compounds (C: $270 \mathrm{~nm}$; D: $340 \mathrm{~nm}$ ), and mixed standard substances (E: 270 $\mathrm{nm}$ ); 7 standard substances were completely detected at $270 \mathrm{~nm}$, so $340 \mathrm{~nm}$ was not done) (1: Rutin; 2: Phillyrin; 4: Chlorogenic acid; 5: Caffeic acid; 8: Forsythiaside A; 9: Hesperidin; 11: Phillygenin).

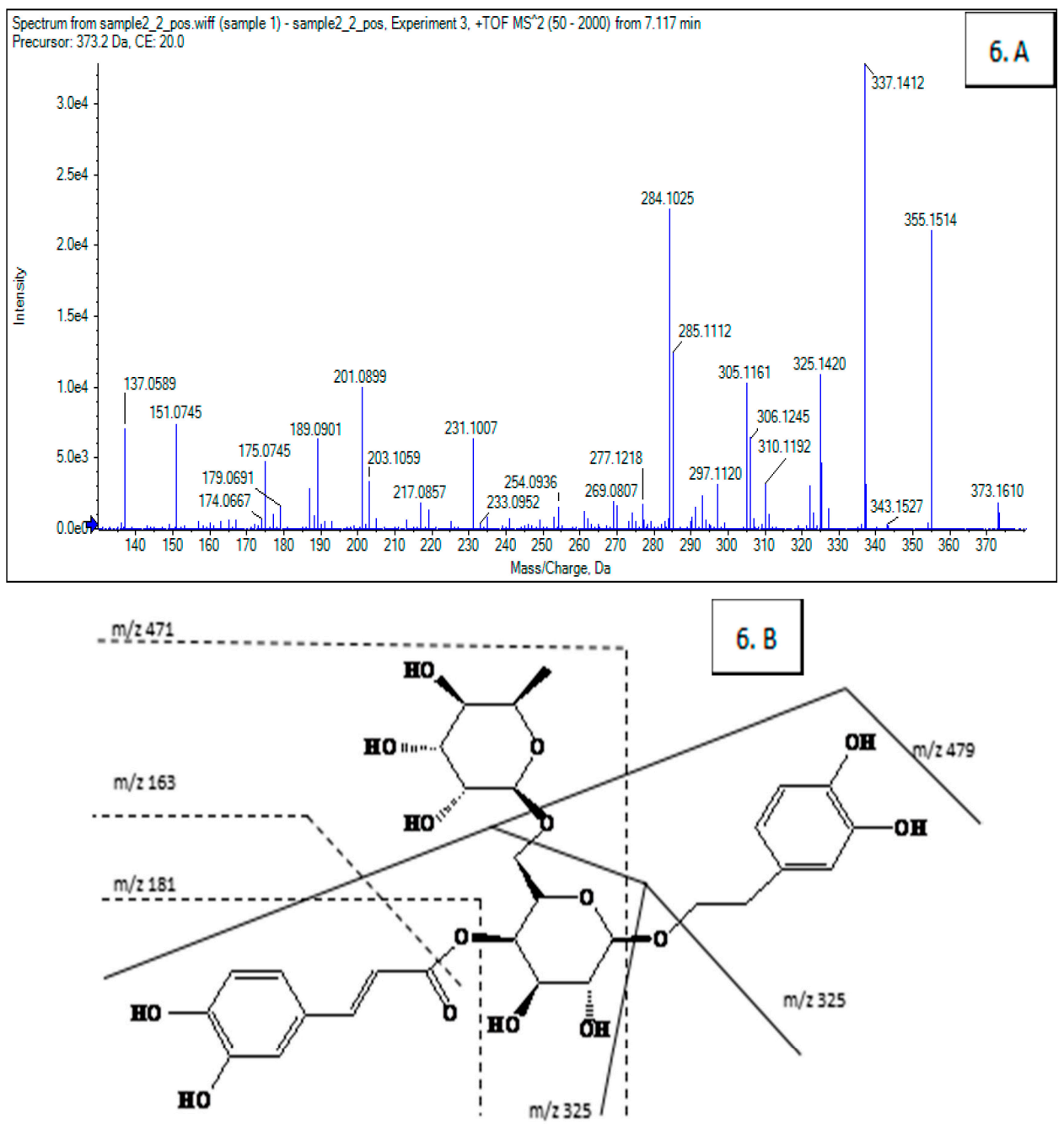

Figure 2. Cont. 

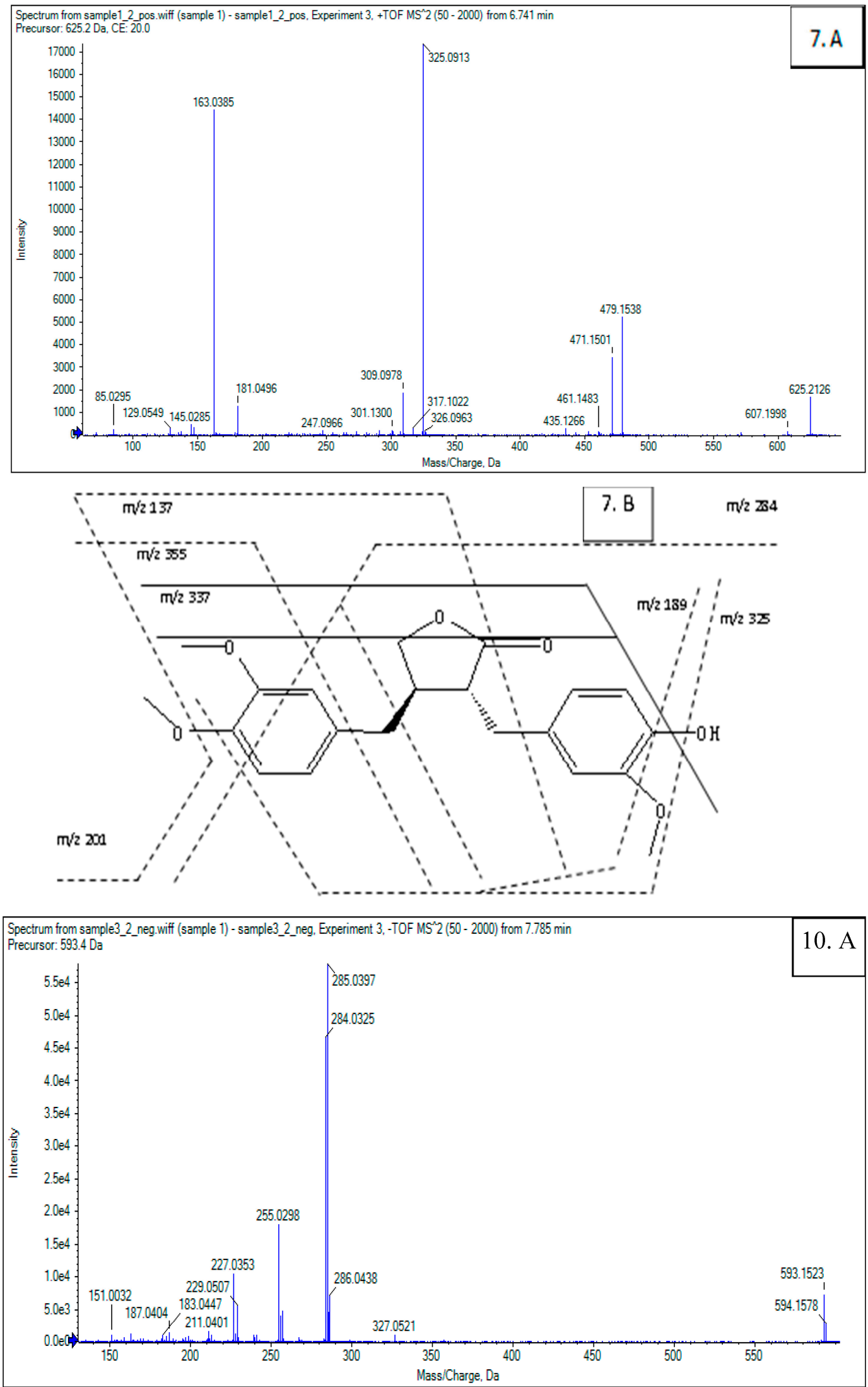

Figure 2. Cont. 


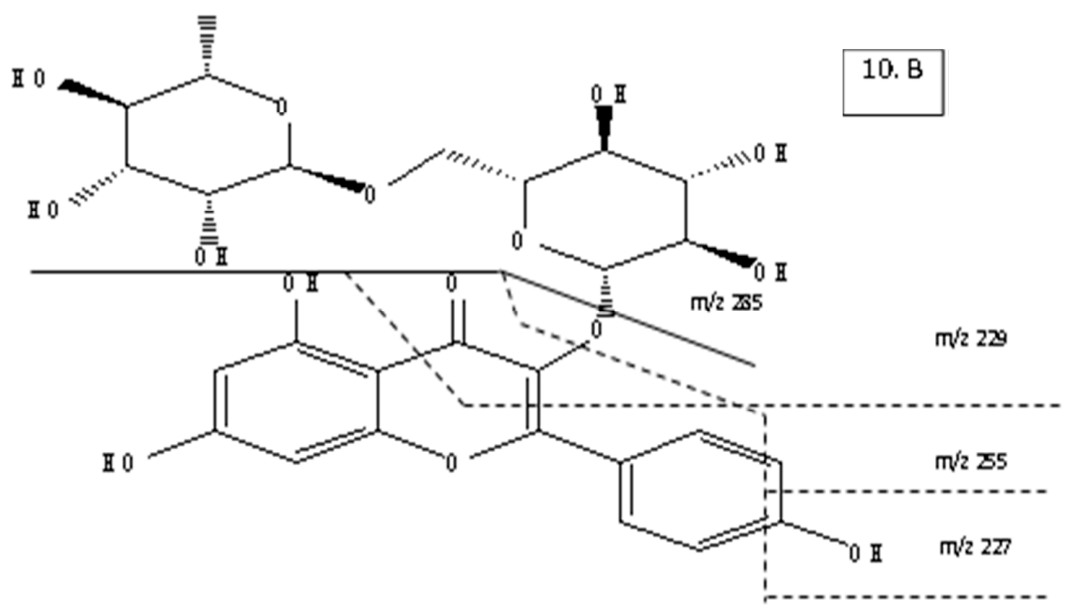

Figure 2. MS2 spectra (A) and fragmentation schemes (B) of Compounds 6, 7, and 10 of F. suspensa leaves. (6: Isomer of forsythiaside A; 7: Arctigenin; 10: Kaempferol-3-O-rutinose).

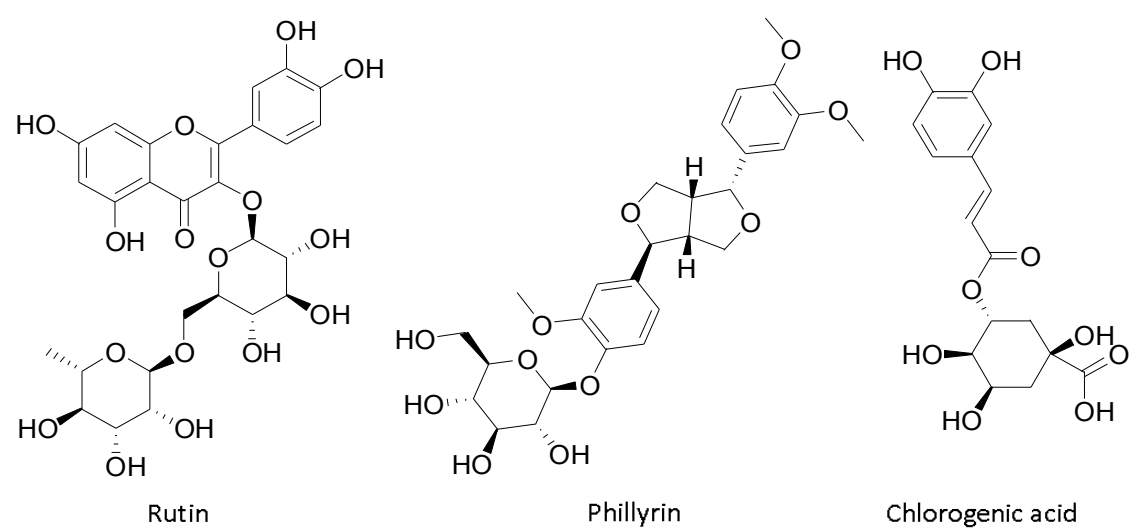

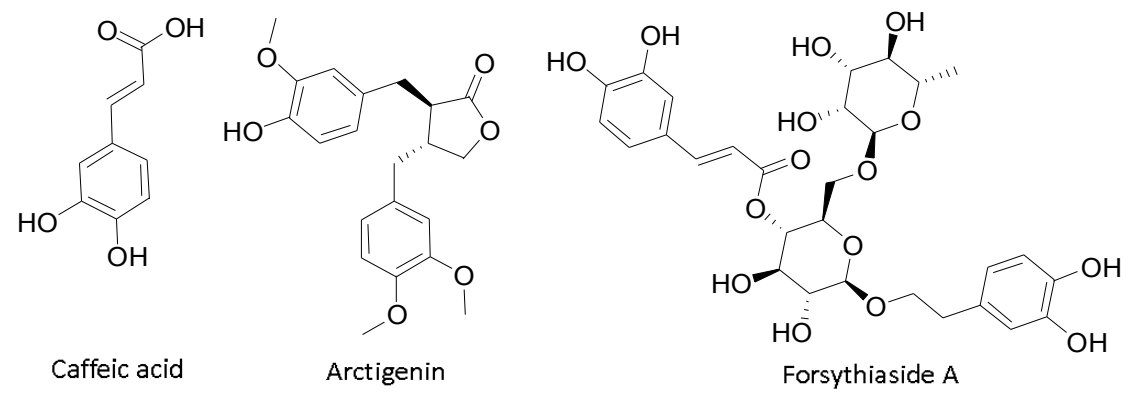

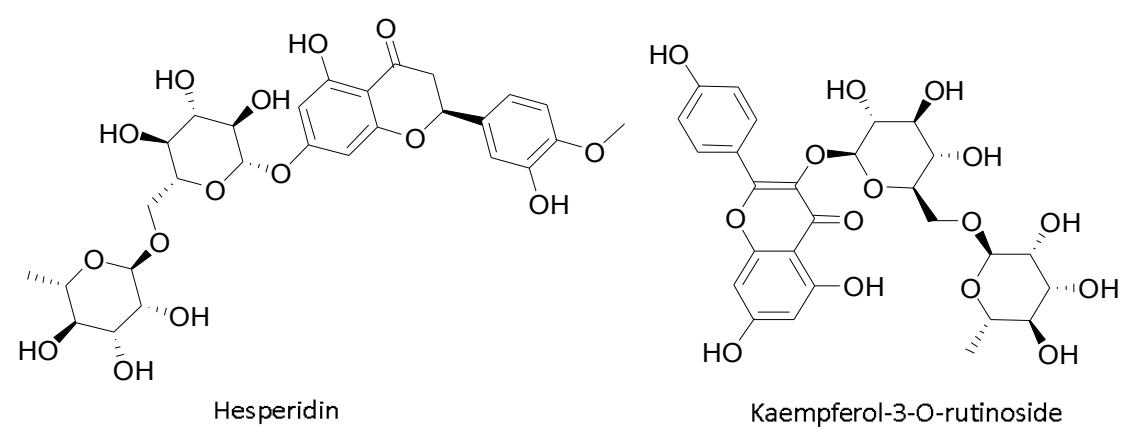

Figure 3. Tentative identified 8 PL ligands from F. suspensa leaves: Rutin (1); Phillyrin (2); Chlorogenic acid (4); Caffeic acid (5); Arctigenin (7); Forsythiaside A (8); Hesperidin (9); Kaempferol-3-O-rutinoside (10). 
Table 2. MS2 analysis of Compounds 6, 7, and 10 of F. suspensa leaves.

\begin{tabular}{cccccc}
\hline Peak No. & $t_{\mathbf{R}}(\mathbf{m i n})$ & UV $(\mathbf{n m})$ & MS $(\mathbf{m} / \mathbf{z})$ & MS2 $(\mathbf{m} / \mathbf{z})$ & Tentative Identification \\
\hline 6 & 9.986 & $216,247,290,330$ & 624 & $479,471,325,309,181,163$ & Isomer of forsythiaside A \\
7 & 15.682 & $208,230,280$ & 372 & $355,337,325,284,201,189,137$ & Arctigenin \\
10 & 26.716 & 265,337 & 594 & $285,255,229,227$, & Kaempferol-3-O-rutinose \\
\hline
\end{tabular}

\subsection{The Detection of Enzyme Inhibitory Activity and Competition Types of All Inhibitors}

Of the eight compounds with identified structures, references [22-25] have shown the PL inhibitory activities of five compounds, including 4 (chlorogenic acid), 5 (caffeic acid), 1 (rutin), 9 (hesperidin), and 10 (kaempferol-3-O-rutinoside) (their inhibitory abilities were not tested in this study; these references only illustrated the success of this experiment system). The PL inhibitory activities of Compounds $\mathbf{7}$ (arctigenin), $\mathbf{8}$ (forsythiaside A), and $\mathbf{2}$ (phillyrin) were detected. The results are shown in Figure 4. Of these eight compounds, the inhibitory effect of kaempferol-3-O-rutinoside on PL was the strongest, with an $\mathrm{IC}_{50}$ value of $2.9 \pm 0.5 \mu \mathrm{mol} / \mathrm{L}$, followed by hesperidin and rutin, with $\mathrm{IC}_{50}$ values of 52.4 and $149 \pm 6.0 \mu \mathrm{mol} / \mathrm{L}$, respectively. The inhibitory effects of caffeic acid, arctigenin, forsythiaside A, and chlorogenic acid were lower. Interestingly, phillyrin exhibited no inhibitory effect on PL, but showed a promoting effect (see Table 3).
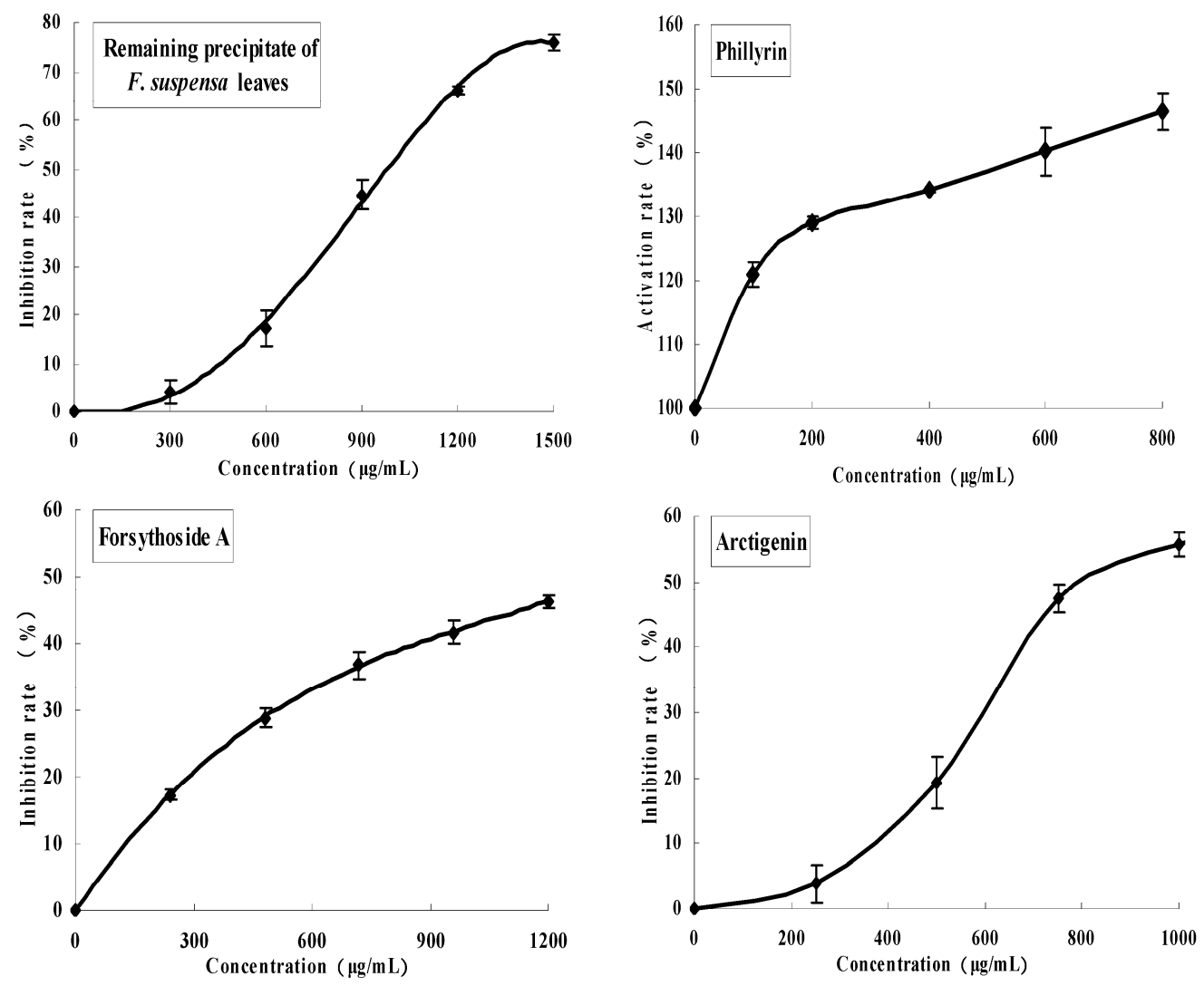

Figure 4. Inhibitive effects of remaining precipitate, phillyrin (Compound 2), forsythioside A (Compound 8), and arctigenin (Compound 7) of F. suspensa leaves on pancreatic lipase.

The standard curve of pNP is shown in Figure 5, the inhibition types of the remaining precipitation and the eight compounds were both detected and are shown in Figure 6. Kaempferol-3-O-rutinoside showed non-competitive inhibition. Rutin, hesperidin, and forsythiaside A showed competitive inhibition (the isomer of forsythiaside A might have the same ability). Caffeic acid, chlorogenic acid, 
and arctigenin exhibited uncompetitive inhibition. Phillyrin was a special compound that showed a competitive promoting effect.

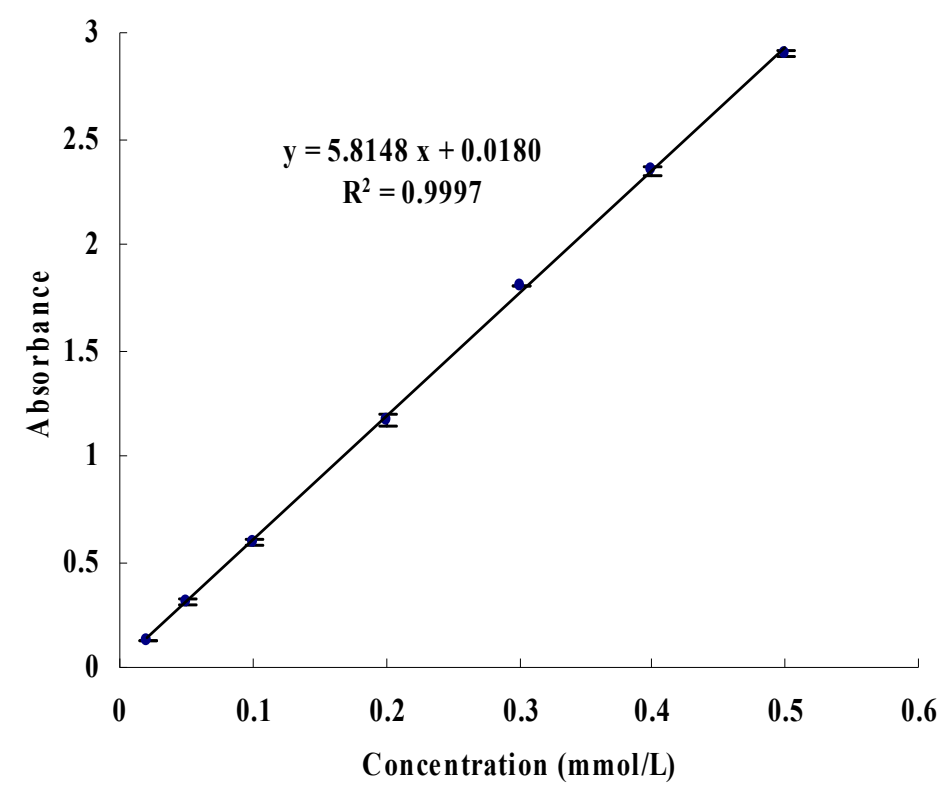

Figure 5. Standard curve of $p$-nitrophenol.
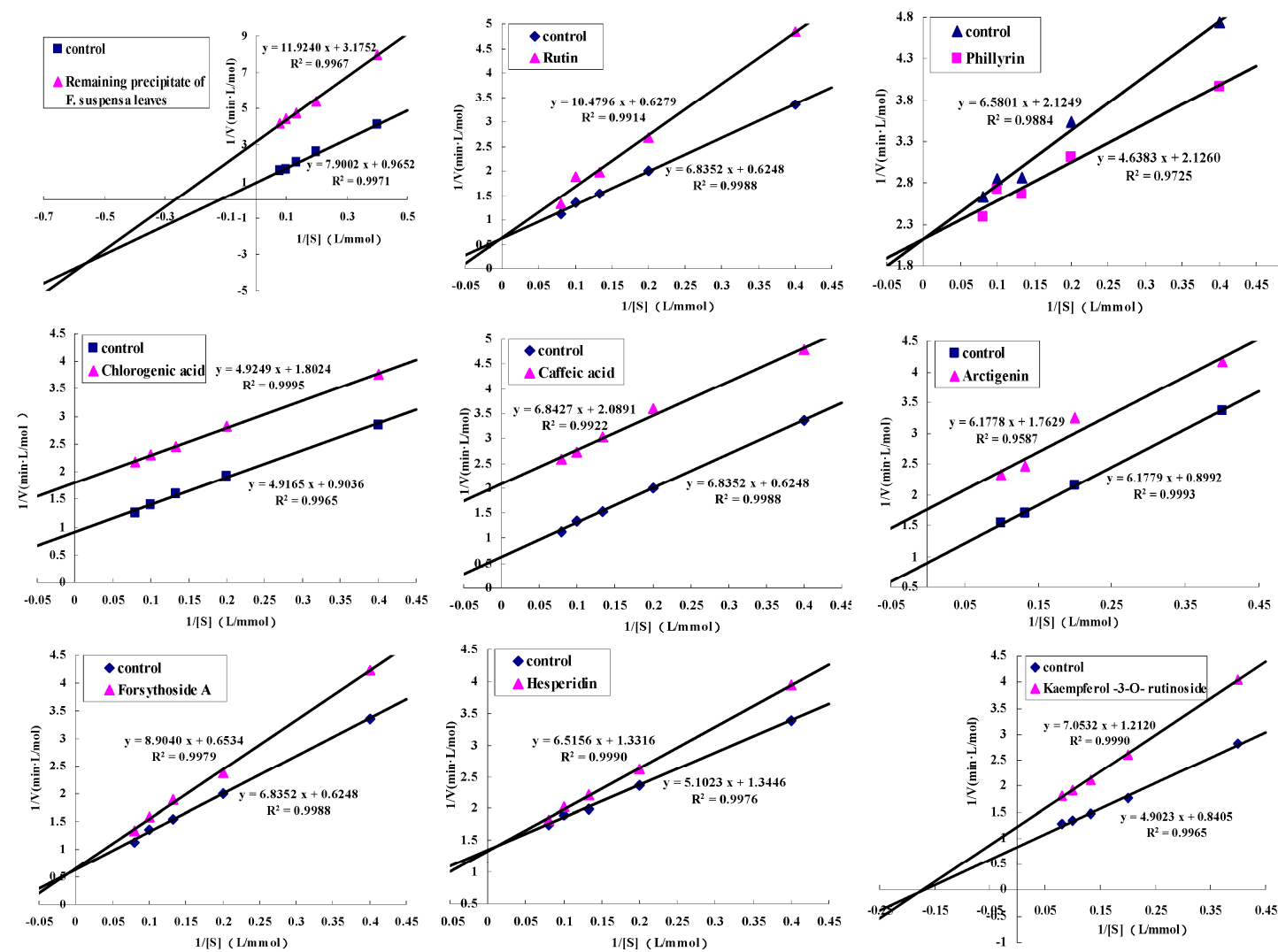

Figure 6. Inhibitive modes of remaining precipitate and screened compounds on pancreatic lipase in $F$. suspensa leaves (remaining precipitate, Rutin (1), Phillyrin (2), Chlorogenic acid (4), Caffeic acid (5), Arctigenin (7), Forsythiaside A (8), Hesperidin (9), Kaempferol-3-O-rutinoside (10)). 
All of the experimental results of the nine ligands are shown in Table 3.

Table 3. Nine pancreatic lipase ligands screened out from F. suspensa leaves $(n=3)$.

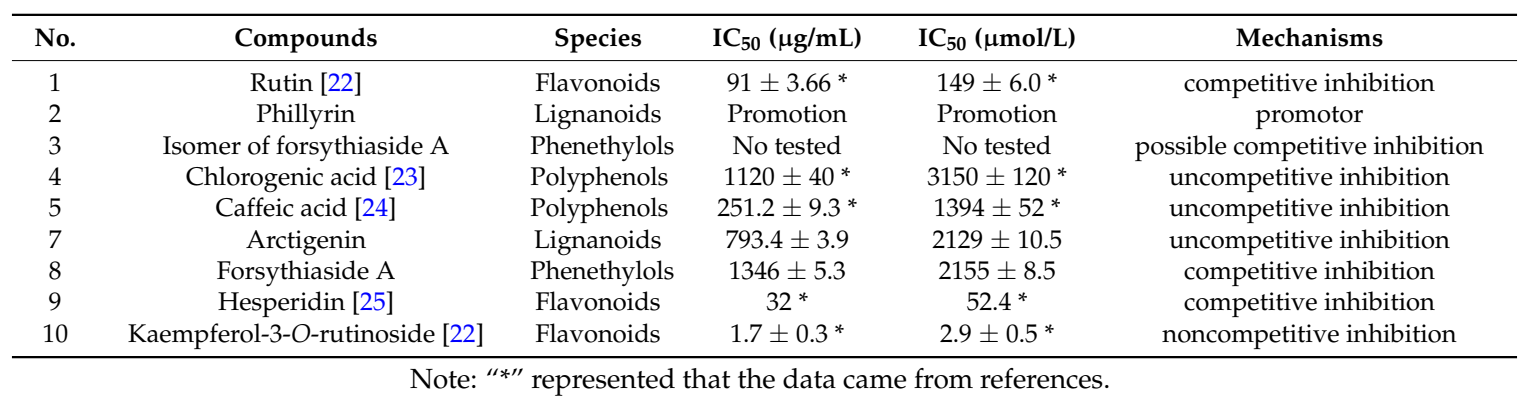

\subsection{Discussion}

In this study, nine ligands that could interact with PL were screened out all at one time by an immobilized enzyme screening model. The reasons are listed as follows: (1) We chose an immobilized enzyme carrier that has as little adsorption ability as possible to avoid nonspecific adsorption; (2) We closed the remaining carboxyl of the magnetic bead surface via small- and little-absorption-ability compounds; (3) We screened for a higher active subfraction; (4) We chose a suitable eluent and denaturant, and their concentration; (5) We chose a suitable HPLC mobile phase and elution gradient to obtain a smooth baseline and good separation effects; (6) We scanned a full wavelength of the screened sample and then chose suitable HPLC detection wavelengths. Put simply, using these methods can lead to compounds with less nonspecific absorption and with more specific absorption.

In this study, two new PL inhibitors, arctigenin and forsythiaside A, were found. Kaempferol-3-O-rutinoside, which could not be found from $F$. suspensa before, was screened out for the first time, and its $\mathrm{IC}_{50}$ notably reached $2.9 \pm 0.5 \mu \mathrm{mol} / \mathrm{L}$; moreover, phillyrin, as a promotor of PL was also found. The isomer of forsythiaside A and compound 6 need to be isolated and identified.

\section{Materials and Methods}

\subsection{Materials}

The following materials were acquired and used: Orlistat (Chongqing Pharscin Pharmaceutical Group Co., Ltd., Chongqing, China); carboxyl-terminated magnetic beads $(10 \mathrm{mg} / \mathrm{mL}, 1 \mu \mathrm{m})$ (Allrun, Shanghai, China); F. suspensa leaves (Shanxi University campus, Taiyuan, China); $\mathrm{PL}, \mathrm{N}$-3-dimethylaminopropyl- $\mathrm{N}^{\prime}$-ethyl-carbodiimide $\mathrm{HCl}$ (EDC), $\mathrm{N}$-hydroxysuccinimide (NHS), 2-(N-morpholino) ethanesulfonic acid (MES), Tween 20, Tris and glycine (Gly) (Solarbio, Beijing, China); $p$-nitrophenyl butyrate $(p N P B)$, and $p$-nitrophenol $(p N P)$ (Sigma-Aldrich, St. Louis, MO, USA); standard compounds, including chlorogenic acid, caffeic acid, forsythiaside A, rutin, hesperidin, and phillyrin (Must Bio-Technology Co., Ltd., Sichuan, China); HPLC-grade methanol and 96-well microtiter plates (Fisher Scientific, Shanghai, China). Other reagents were analytically pure. All solutions were prepared by ultra pure water.

\subsection{Preparation of the Subfractions of F. suspensa Leaves}

The leaves of F. suspensa (Thunb.) Vahl were collected in the campus of Shanxi University in July 2014 and identified by Prof. Liwei Zhang, Institute of Molecular Science, Shanxi University, Taiyuan 030006, China. A voucher specimen (no. SXTY-05337) had been deposited at the Herbarium of Institute of Molecular Science, Shanxi University, China. First, the dried leaf powder (300 g) was added to $3 \mathrm{~L}$ of $70 \%$ ethanol, reflux was extracted for $1 \mathrm{~h}$, and then the hot extraction solution was filtered. The extraction and filtration process was repeated twice. Second, the filtrates were concentrated to 
$500 \mathrm{~mL}$ (note: there was some water in $500 \mathrm{~mL}$ of concentrated liquid; if it was dried, its weight was $99.29 \mathrm{~g}$, and the yield was $33.09 \%$ ). Third, petroleum ether and ethyl acetate was used for extraction sequentially $(1: 1, v / v$, twice) from the cooled concentrated liquid. Fourth, ethanol and water were employed to dissolve the remaining mixture sequentially $(1: 10, v / v$, once) (note: due to a long extraction time, repeated extraction, and hot filtration, the $70 \%$ ethanol extraction contained various substances that could not be completely dissolved in cold ethanol and could be divided into five parts: an easily soluble part in cold petroleum ether, an easily soluble part in cold ethyl acetate, a soluble part in cold ethanol, a soluble part in cold water, and a final water-insoluble part). Finally, the corresponding subfractions and the final water-insoluble substance were dried. The petroleum ether extraction, ethyl acetate extraction, ethanol-soluble substance, water-soluble substance, and the final water-insoluble substance yields were $4.46 \%, 9.13 \%, 15.04 \%, 2.43 \%$, and $1.81 \%$, respectively.

\subsection{Determination of Enzyme Inhibitory Activity of All Subfractions}

A PL solution $(100 \mu \mathrm{L}, 0.5 \mathrm{mg} / \mathrm{mL})$ was placed in a $1.5 \mathrm{~mL}$ centrifuge tube, and $20 \mu \mathrm{L}$ of sample solution was then added. A Tris- $\mathrm{HCl}$ buffer ( $\mathrm{pH} 7.5$ ) was added to $900 \mu \mathrm{L}$, mixed, and incubated for $15 \mathrm{~min}$ at $37^{\circ} \mathrm{C}$. A $p$ NPB solution $(100 \mu \mathrm{L}, 10 \mathrm{mmol} / \mathrm{L})$ was added to the solution, which was oscillated and then quickly transferred to a 96-well microtiter plate. Finally, the change rate $\mathrm{K}$, which is the change of absorbance of the solution at $400 \mathrm{~nm}$ over $15 \mathrm{~min}$, was measured with a microplate reader. The inhibition rate was calculated as follows:

Inhibition rate $(\%)=(\mathrm{K}$ normal value $-\mathrm{K}$ experimental value $) / \mathrm{K}$ normal value $\times 100 \%$.

In the reaction system, $\mathrm{PL}, p \mathrm{NPB}$, and DMSO were used as a normal group, $\mathrm{PL}, p \mathrm{NPB}$, and orlistat (DMSO solution) as a positive control group, and PL, $p$ NPB, and inhibitor (DMSO solution) as an experimental group. $\mathrm{K}$ normal value $=(p \mathrm{NP}$ absorbance (after $15 \mathrm{~min})-p \mathrm{NP}$ absorbance $(0 \mathrm{~min})) / 15$, namely the rate of enzyme reaction, in normal group at $400 \mathrm{~nm}$, and $\mathrm{K}$ experimental value was the same, but in experimental group. $p$ NP was the product of $p$ NPB catalyzed by PL. 0 min meant the beginning of absorbance detection by Microplate Reader. Each experiment was repeated three times.

The inhibition rates of all subfractions on PL were measured at $1.5 \mathrm{mg} / \mathrm{mL}$.

\subsection{Screening of Inhibitors in the Subfraction with the Highest Inhibitory Activity}

Synthesis, characterization, stability, specificity, and repeatability of immobilized pancreatic lipase were published in [14].

The subfraction with the highest inhibitory activity was used to screen the PL inhibitors. The process was similar to the validation of immobilized PL. A Thermo Scientific Hypersil Gold C18 column $(4.6 \mathrm{~mm} \times 250 \mathrm{~mm}, 5 \mu \mathrm{m})$ was used for HPLC separation and detection. The detection wavelength was 270 and $340 \mathrm{~nm}$. The column temperature and flow rate were $25^{\circ} \mathrm{C}$ and $0.8 \mathrm{~mL} / \mathrm{min}$, respectively. The mobile phase was composed of methanol (A) and $0.3 \%$ acetic acid solution (B). The gradient elution was set as follows: 0-8 $\mathrm{min}, 30-33 \%$ A; $8-24 \mathrm{~min}, 33-40 \%$ A; $24-39 \mathrm{~min}, 40-48 \%$ A; and $39-55 \mathrm{~min}, 48-64 \%$ A.

\subsection{Identification of Screened-Out Compounds}

Comparison with the HPLC chromatograms of a mixture of standard compounds: Suitable amounts of chlorogenic acid, caffeic acid, forsythiaside A, rutin, hesperidin, phillyrin, and forsythiaside standards were dissolved in methanol and detected according to the aforementioned chromatographic conditions.

LC-MS identification: The compounds that were not matched with HPLC chromatograms of a mixture of standard compounds were separated and tentatively identified by LC-MS. 


\subsection{Determination of Enzyme Inhibitory Activity and Competition Types of Screened-Out Compounds}

The detection method of enzyme inhibitory activity was similar to a determination of enzyme inhibitory activity of all subfractions. The test method of competition types was that $p$ NP standard solution with concentrations of $0.02,0.05,0.1,0.2,0.3,0.4$, and $0.5 \mathrm{mmol} / \mathrm{L}$ was precisely prepared. When the standard curve was drawn, a PL solution $(0.5 \mathrm{mg} / \mathrm{mL})$ and the inhibitor solution with concentrations close to $\mathrm{IC}_{50}$ were prepared. The solution with no inhibitor was regarded as the control group, and solutions with an addition of inhibitor was regarded as the experimental group. The enzyme reaction rates were determined when the substrate concentrations were $0.25,0.50,0.75$, 1.00, and $1.25 \mathrm{mmol} / \mathrm{L}$. The map was drawn by Lineweaver-Burk double reciprocal plot method.

\section{Conclusions}

Using the appropriate methods, more active ingredients can be simply and quickly screened out all at one time from a complex natural product system. In conclusion, F. suspensa leaves contain numerous inhibitors of pancreatic lipase. A potential use of this tea for weight loss remains to be shown in pharmacological studies.

Acknowledgments: This work was supported by the Key Technology Research of Traditional Chinese Medicine Modernization of Shanxi Province (Zhendong Special) (2014ZD0304).

Author Contributions: T.C. and L.Z. conceived and designed the experiments; Y.L. performed the experiments; T.C., Y.L. and L.Z. analyzed the data; T.C. and L.Z. contributed reagents/materials/analysis tools; T.C. wrote the paper.

Conflicts of Interest: The authors have declared no conflict of interest.

\section{References}

1. Report of a WHO Consultation. Obesity: Preventing and Managing the Global Epidemic; World Health Organization: Geneva, Switzerland, 2000.

2. Haslam, D.W.; James, W.P.T. Obesity. Lancet 2005, 366, 1197-1209. [PubMed]

3. World Health Organization. Available online: http://www.who.int/mediacentre/factsheets/fs311/en/ index.html (accessed on June 2016).

4. Ezzati, M.; Lopez, A.D.; Rodgers, A.; Vander Hoorn, S.; Murray, C.J. Comparative Risk Assessment Collaborating Group. Selected major risk factors and global and regional burden of disease. Lancet 2002, 360, 1347-1360. [PubMed]

5. U.S. Department of Health and Human Services. Available online: http://www.fda.gov/ (accessed on June 2016).

6. Baretic, M. Targets for medical therapy in obesity. Digest. Dis. Sci. 2012, 30, 168-172.

7. Derosa, G.; Maffioli, P. Anti-obesity drugs: A review about their effects and their safety. Expert Opin. Drug Saf. 2012, 11, 459-471. [PubMed]

8. Yun, J.W. Possible anti-obesity therapeutics from nature-A review. Phytochemistry 2010, 71, $1625-1641$. [PubMed]

9. Roh, C.; Jung, U. Screening of crude plant extracts with anti-obesity activity. Int. J. Mol. Sci. 2012, 13, 1710-1719. [PubMed]

10. Birari, R.B.; Bhutani, K.K. Pancreatic lipase inhibitors from natural sources: Unexplored potential. Drug Discov. Today 2007, 12, 879-889. [PubMed]

11. De la Garza, A.L.; Milagro, F.I.; Boque, N.; Campion, J.; Martinez, J.A. Natural inhibitors of pancreatic lipase as new players in obesity treatment. Planta. Medica. 2011, 77, 773-785. [PubMed]

12. Del Castillo-Santaella, T.; Maldonado-Valderrama, J.; Cabrerizo-Vilchez, M.A.; Rivadeneira-Ruiz, C.; Rondon-Rodriguez, D.; Galvez-Ruiz, M.J. Natural inhibitors of lipase: Examining lipolysis in a single droplet. J. Agric. Food Chem. 2015, 63, 10333-10340. [PubMed]

13. Tao, Y.; Zhang, Y.F.; Cheng, Y.Y.; Wang, Y. Rapid screening and identification of $\alpha$-glucosidase inhibitors from mulberry leaves using enzyme-immobilized magnetic beads coupled with HPLC/MS and NMR. Biomed. Chromatogr. 2013, 27, 148-155. [PubMed] 
14. Chen, T.-G.; Wei, Y.-R.; Zhang, L.-W. Screening and Identification of pancreatic lipase inhibitors in polygonum cuspidatum with enzyme-immobilized magnetic nanoparticles and LC-MS/MS. Nat. Prod. Res. Dev. 2017, 29, 198-205.

15. Qu, H.H.; Zhang, Y.M.; Wang, Y. Antioxidant and antibacterial activity of two compounds (forsythiaside and forsythin) isolated from Forsythia suspensa. J. Pharm. Pharmacol. 2008, 60, 261-266. [PubMed]

16. Zhang, G.G.; Song, S.J.; Ren, J.; Xu, S.X. A new compound from Forsythia suspensa (Thunb.) Vahl with antiviral effect on RSV. J. Herb. Pharmacol. 2002, 2, 35-40.

17. Dai, S.J.; Ren, Y.; Shen, L.; Zhang, D.W. New alkaloids from Forsythia suspensa and their anti-inflammatory activities. Planta Med. 2009, 75, 375-377. [PubMed]

18. Kang, W.Y.; Wang, J.M. In vitro antioxidant properties and in vivo lowering blood lipid of Forsythia suspense leaves. Med. Chem. Res. 2010, 19, 617-628.

19. Yuda, N.; Tanaka, M.; Suzuki, M.; Asano, Y.; Ochi, H.; Iwatsuki, K. Polyphenols extracted from black tea (Camellia sinensis) residue by hot-compressed water and their inhibitory effect on pancreatic lipase in vitro. J. Food Sci. 2012, 77, H254-H261. [PubMed]

20. Nakai, M.; Fukui, Y.; Asami, S.; Toyoda-Ono, Y.; Iwashita, T.; Shibata, H.; Mitsunaga, T.; Hashimoto, F.; Kiso, Y. Inhibitory effects of oolong tea polyphenols on pancreatic lipase in vitro. J. Agric. Food Chem. 2005, 53, 4593-4598. [PubMed]

21. Wu, X.; He, W.; Yao, L.; Zhang, H.; Liu, Z.; Wang, W.; Ye, Y.; Cao, J. Characterization of binding interactions of (-)-epigallocatechin-3-gallate from green tea and lipase. J. Agric. Food Chem. 2013, 61, 8829-8835. [PubMed]

22. Habtemariam, S. Antihyperlipidemic components of cassia auriculata aerial parts: Identification through in vitro studies. Phytother. Res. 2013, 27, 152-155.

23. Narita, Y.; Iwai, K.; Fukunaga, T.; Nakagiri, O. Inhibitory activity of Chlorogenic acids in decaffeinated green coffee beans against porcine pancreas lipase and effect of a decaffeinated green coffee bean extract on an emulsion of olive oil. Biosci. Biotechnol. Biochem. 2012, 76, 2329-2331. [PubMed]

24. Cai, S.B.; Wang, O.; Wang, M.Q.; He, J.F.; Wang, Y.; Zhang, D.; Zhou, F.; Ji, B.P. In vitro inhibitory effect on pancreatic lipase activity of subfractions from ethanol extracts of fermented Oats (Avena sativa L.) and synergistic effect of three phenolic acids. J. Agric. Food Chem. 2012, 60, 7245-7251. [PubMed]

25. Kawaguchi, K.; Mizuno, T.; Aida, K.; Uchino, K. Hesperidin as an inhibitor of lipases from porcine pancreas and pseudomonas. Biosci. Biotechnol. Biochem. 1997, 61, 102-104. [PubMed]

Sample Availability: Samples of the compounds rutin, phillyrin, chlorogenic acid, caffeic acid, forsythiaside A, hesperidin, phillygenin are available from the authors. 